\title{
(2) OPEN ACCESS \\ Chemotherapy decision-making in advanced lung cancer: a prospective qualitative study
}

Annmarie Nelson, ${ }^{1}$ Mirella Longo (1) , ${ }^{1}$ Anthony Byrne, ${ }^{1}$
Stephanie Sivell, ${ }^{1}$ Simon Noble (D) , 'Jason Lester, ${ }^{2}$ Lesley Radley, ${ }^{3}$
David Jones, ${ }^{3}$ Catherine Sampson, ${ }^{1}$ Despina Anagnostou ${ }^{1}$

- Additional material is published online only. To view please visit the journal online (http://dx.doi.org/10.1136/ bmjspcare-2020-002395).

${ }^{1}$ Marie Curie Palliative Care Research Centre, School of Medicine, Cardiff University, Cardiff, UK

${ }^{2}$ Velindre Cancer Centre, Cardiff UK

${ }^{3}$ Cardiff University, Cardiff, UK

\section{Correspondence to}

Professor Annmarie Nelson,

Marie Curie Palliative Care

Research Centre, School of

Medicine, Cardiff University, Cardiff cf14 4ys, South

Glamorgan, UK;

nelsona9@Cardiff.ac.uk

Received 4 May 2020

Revised 28 June 2020

Accepted 29 June 2020
D Check for updates

(C) Author(s) (or their employer(s)) 2020. Re-use permitted under CC BY-NC. No commercial re-use. See rights and permissions. Published by BMJ.

To cite: Nelson $A$, Longo $M$, Byrne $\mathrm{A}$, et al. BMJ Supportive \& Palliative Care Epub ahead of print: [please include Day Month Year]. doi:10.1136/

bmjspcare-2020-002395

\section{ABSTRACT}

Objective To study how treatment decisions are made alongside the lung cancer clinical pathway.

Methods A prospective, multicentre, multimethods, five-stage, qualitative study. Mediated discourse, thematic, framework and narrative analysis were used to analyse the transcripts.

Results 51 health professionals, 15 patients with advanced lung cancer, 15 family members and 18 expert stakeholders were recruited from three UK NHS trusts. Multidisciplinary team (MDT) members constructed treatment recommendations around patient performance status, pathology, clinical information and imaging. Information around patients' social context, needs and preferences were limited. The provisional nature of MDTs treatment recommendations was not always linked to future discussions with the patient along the pathway, that is, patients' interpretation of their prognosis, treatment discussions occurring prior to seeing the oncologist. This together with the rapid disease trajectory placed additional stress on the oncologist, who had to introduce a different treatment option from that recommended by the MDT or patient's expectations. Palliative treatment was not referred to explicitly as such, due to its potential for confusion. Patients were unaware of the purpose of each consultation and did not fully understand the non-curative intent of treatment pathways. Patients' priorities were framed around social and family needs, such as being able to attend a family event.

Conclusion Missed opportunities for information giving, affect both clinicians and patients; the pathway for patients with non-small cell lung cancer focuses on clinical management at the expense of patient-centred care. Treatment decisions are a complex process and patients draw conclusions from healthcare interactions prior to the oncology clinic, which prioritises aggressive treatment and influences decisions.

\section{INTRODUCTION}

New developments in immunotherapy offer promising treatment for advanced lung cancer. ${ }^{1}$ However, a considerable number of patients with non-small cell lung cancer (NSCLC) present with locally advanced or metastatic disease and are currently unsuitable for curative treatment with a median survival of 6-8 months. ${ }^{2}$ The fear of destroying hope places a huge burden on the clinicians who may resort to the treatment imperative; ${ }^{3}$ patients' limited understanding of treatment with palliative intent ${ }^{4}$ further hinders the patients' opportunity to consider all available options and to make an informed decision. Treatment decisions become time bound, complex and require information and awareness for both patients and clinicians.

Chemotherapy with palliative intent is used for symptom control and to improve quality of life; however, systemic anticancer treatment (SACT) may have unintentionally detrimental effects. For patients in advanced stage, including NSCLC, SACT can increase early treatment-related mortality, ${ }^{6-10}$ toxicities, emergency and inpatient admissions ${ }^{11}$ and affect quality of life, even in patients with good performance status. ${ }^{12}$ Around 10\% of patients with lung cancer are dying within 30 days of SACT and this is arguably overly aggressive care. The urgency of administering treatments, in an attempt to mitigate deterioration, can also result in underpreparation of patients and families at the end of life ${ }^{1314}$ and patients become less likely to die in their preferred place of death. ${ }^{15}$ Documentation of end 
of life discussions and discontinuation of treatment is rare; ${ }^{16}{ }^{17}$ however, early links with palliative care teams improve the timing of final chemotherapy administration and transition to hospice services. ${ }^{18-20}$ Patients opting out of $\mathrm{SACT}^{21}$ show greater acceptance of terminal status, focus on the present and on time with family.

The WHO places responsiveness and participation as key to achieving integrated people-centred health services and advocates care in respect of people's preferences. ${ }^{22}$ Clinical pathways aim to support decisions along the treatment pathway; however, in oncology, these seem to focus on when to start anticancer treatment-not when to stop. Studying the treatment decision-making process, alongside the patient clinical pathway, helps to understand the interaction of factors at different levels and how these influence the success or the failure of patient-centred care in the context of these very sensitive consultations. This study aimed to understand how palliative chemotherapy treatment decisions are determined and what intervention could support Patients with NSCLC and clinicians when considering AntiCancer Therapy (the PACT study). The paper adheres to Consolidated criteria for Reporting Qualitative research guidelines (online supplementary 1 ; online supplementary table e1).

\section{METHODS}

The PACT study was a five-stage, prospective, qualitative multicentre, multimethods study. The original protocol $^{23}$ was adhered to with the exception of using narrative analysis in place of interpretative phenomenological analysis as clear narratives emerged in the data. A conceptual framework was built around study aim and objectives (figure 1).

\section{Participants}

A stakeholder approach was used to purposively sample study participants. For phases 1-4, clinicians and patients were recruited from three hospitals set within three University Health Boards in Wales. UK experts with an interest in lung cancer were invited to the expert consensus event. The data collected

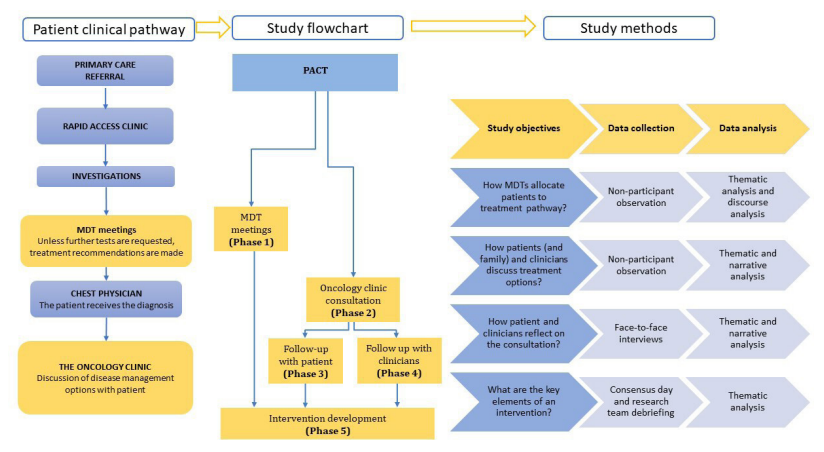

Figure 1 Patients with non-small cell lung cancer and clinicians when considering AntiCancer Therapy (PACT) conceptual framework. MDT, multidisciplinary team. throughout phases 2-4 included the same patient/ doctor dyad. Patient and public involvement (PPI) was incorporated throughout the study and in accordance with the national standards.

\section{Approach and consent}

Patients with a diagnosis of NSCLC and due to attend their first appointment with the oncologist were eligible to be part of the study. Multidisciplinary team (MDT) members were recruited and consented first. Clinicians identified eligible participants and provided them with an information sheet and a cover letter which included a reply slip where they would specify their contact details if they wished to be contacted about the study. Consent from clinicians, patients, companions and experts attending the consensus event was taken by the researchers (CS, DS, ML). Audio recording and fields notes were used throughout.

\section{Data analysis}

Inductive thematic analysis was used initially to aid understanding of the data before specific methodologies were overlaid. Principles of mediated discourse analysis were applied to MDT meetings to understand team dynamics and working practices, the role played by key MDT members and factors influencing allocation of patients to treatment pathways. Narrative analysis was used to study the consultations between the patient/companion and clinician and follow-up faceto-face interviews. Narrative analysis highlighted the priorities of the participants in single consultations; it also enabled the decision-making trajectory to be modelled through a generic consultation with priorities for each participant indicated at each phase. The OPTION (Observing PaTient InvOlvemeNt) tool ${ }^{24}$ was used as a consultation process framework to assess how clinicians involved patients in consultations under key clinical domains (online supplementary 2; online supplementary table e2); this was reflected in the interview guide (online supplementary 4). Phase 5 drew together expert stakeholders and provided the platform where information from phases 1 to 4 could be shared, questioned and prioritised by all parties. Group discussions were held and covered seven areas of enquiry (eg, patients' preparedness; online supplementary 4, online supplementary table e3). The composition of groups ensured that while the focus was on the patient, the intervention would receive input from carers and health professionals at each stage of the clinical pathway. A deductive thematic analysis (coding tree focus group 3-online supplementary 5 , online supplementary tables e4-e10) of the group discussions was initially organised around responses to the areas of enquiry and then transformed into a series of statements of suggested practice attached to the clinical pathway. Data saturation was determined by the researchers. NVIVO (V.12) was used to organise the data. 


\section{Research team reflexivity}

The research team comprised clinical oncology consultants, palliative care consultants, patient representatives and experienced postdoctoral researchers with expertise in healthcare research, decision-making, ethnography, discourse analysis and narrative analysis. This variety helped to balance knowledge and assumptions, personal experiences and biases towards aims of treatment, or the dynamic relationships of patients and healthcare professionals. Data collection was undertaken by experienced and trained researchers (DS, CS, ML) not associated with patients or clinical sites.

\section{RESULTS}

Between March 2015 and January 2017, a total of 99 participants were recruited into the study (online supplementary 6; online supplementary table e11). Participants who declined to take part were not recorded.

\section{PPI involvement}

One public contributor (PC) contributed to the development of the research proposal. During the study, two PCs commented on the: information available to patients and their families; jargon used at MDT meetings; and patient recruitment strategies. They took part in the consensus day. They read a sample of anonymised transcripts of patient-clinician consultations and contributed to validate, interpret and contextualise the findings. The PCs continue to be involved in the dissemination of the study results and have agreed to join the follow-up study.

\section{Lung cancer MDT meetings}

Each MDT meeting followed local site routines; however, the primary purpose was to provide accurate diagnosis and recommend treatment pathways. Meetings usually included a chest physician, an oncologist, a radiologist, a pathologist, a lung clinical nurse specialist (CNS) and an MDT coordinator. Palliative care consultants were not always present. Patients were primarily represented through technology (figure 2). Patient-focused clinical and social information was limited.

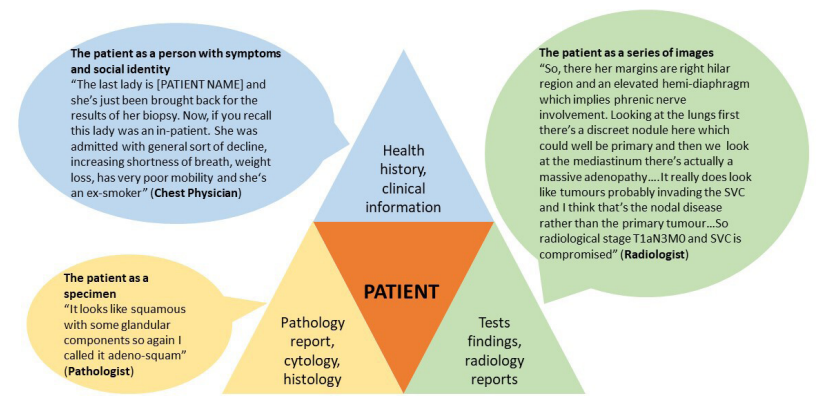

Figure 2 Presenting patient information at the multidisciplinary team meeting.
Radiology and pathology reports were the key references for making treatment recommendations, with the chest physician and the oncologist making key contributions to the decision-making discussions. Performance status was significant in moderating discussion of treatment options. However, treatment decisions were sometimes based on a historical performance status (quotation 1-online supplementary 7, online supplementary table e12).

The provisional nature of a treatment recommendation was not always clearly linked to future discussions with the patient at identified key points on the pathway, that is, considering what the chest physician would discuss and how the patient's interpretation of their prognosis and any treatment discussions would be conveyed to the oncologist.

\section{Oncology clinic}

Four themes emerged from the analysis of the oncology consultation and follow-up interviews: process and priorities were key themes influencing the choice/availability of treatment options and decision-making in the oncology consultation; and the concept of palliative treatment intent and prognosis were key challenges to communication of options. The intersection between these four themes influenced the explicit discussion of options and terminal prognoses and gave insights into the uncertainty for patients around treatment options (quotation 2-online supplementary 7, online supplementary table e12).

\section{Process}

Patients were generally unaware that the oncology consultation was a treatment decision-making event and carried expectations about their treatment plan originating earlier by prior events on the clinical pathway (for instance, discussion with the chest physician) (quotations 3 and 4-online supplementary 7, online supplementary table e12). This suggests a series of missed opportunities for 'preparedness' from both parties.

\section{Priorities}

The oncology consultation evolved over several stages; at each stage, each stakeholder held specific priorities (figure 3).

Patients focused on relationships, placing their disease in the context of everyday life and were concerned with what would happen next. Treatment context was focused on external priorities, such as being able to attend a special family event, and existing relationships with health professionals, all of which influenced their perception and knowledge of a particular treatment option (quotation 5-online supplementary 7, online supplementary table e12). Key issues were challenges for successful communication of their priorities, expertise and constancy within health 


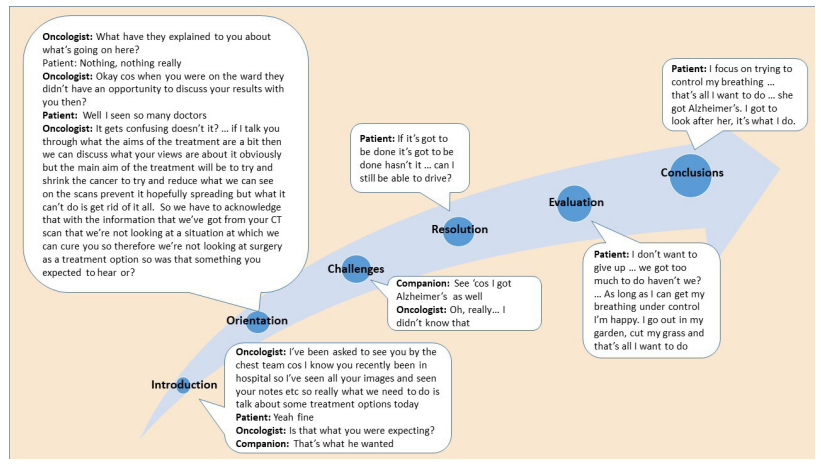

Figure 3 Example of narrative analysis applied to the oncology consultation.

professional relationships, and the need for adequate support to live with the uncertainty of their disease trajectory.

Oncologists were concerned with establishing rapport, were aware of time pressures and followed their own established structure. Performance status description alone did not provide the necessary contextual information for the oncologist who often had to re-evaluate the patient at the time of consultation. Key issues were communicating other treatment options (quotation 6-online supplementary 7, online supplementary table e12), managing uncertainty; particularly where the proposed MDT treatment decision trajectory had changed or where expectations from the chest physician consultation were no longer possible (quotation 7-online supplementary 7, online supplementary table e12).

Companions acted as the patient advocate and their role was to confirm patients' knowledge, needs and preference (quotation 8-online supplementary 7, online supplementary table e12) and provide information on the patient's quality of life. Key issues were preparing the patient to be as well as possible for treatment, having a consistent point of contact with the health professional team, and being aware of potentially different coping styles between patients and carers.

CNSs advocated for the patient perspective and highlighted relationships, patient's home context and priorities, where they were known to them (quotation 9-online supplementary 7, online supplementary table e12). Key issues were the need for ongoing contact, explain terminology and outline treatment practicalities again, and giving the patient opportunities to reflect on their decisions.

\section{Prognosis}

Situations where a patient did not wish to discuss prognosis explicitly presented challenges for the oncologist (quotation 10-online supplementary 7 , online supplementary table e12). Similar challenges arose when there was no formal documentation of how prognosis had been discussed with, and understood by, the patient and carer. Communication skills were key to the confidence of the oncologist in navigating the consultation without directly discussing prognosis.

\section{Palliative intent of treatment}

The most common treatment option offered was palliative chemotherapy, where the word 'palliative' was unspoken and remained implicit in the description (quotation 11 -online supplementary 7 , online supplementary table e12). There was potential for broad interpretation around the possibility of chemotherapy extending life (quotation 12-online supplementary 7, online supplementary table e12), and patient misunderstanding of the purpose as being palliative. However, when palliative was used, this often led to confusion as patients were uncertain about what exactly the oncologist was referring to.

The distinction between palliative treatments perceived as 'active' was significant, which indicated the lack of a positive alternative supportive care option. This was reinforced by oncologists' discussion of their reluctance to use palliative terminology to avoid distress, suggesting ambivalence or lack of understanding around how palliative chemotherapy is presented (quotation 13-online supplementary 7, online supplementary table e12).

\section{Treatment decision around SACT}

Patients opting for SACT without questioning the oncologist had often discussed the proposed treatment option with the chest physician or CNS and had placed their trust in those health professionals. A further deciding factor was where patients felt that their only option was SACT, compounded by the lack of clear definition of what constitutes an active palliative pathway as a viable option.

Opting out of a proposed treatment was challenging for patients; patients did not want 'active treatment', as described by oncologists, for quality of life reasons (quotation 14-online supplementary 7, online supplementary table e12) but were compromised further when oncologists positioned palliative chemotherapy as an option that had to be overtly refused. Table 1 displays the treatment pathways and survival of our study participants.

\section{Consensus day: defining the key components of the intervention}

The findings from phases 1 to 4 set the framework underpinning the design of the intervention. These consistently highlight a lack of 'preparedness' along the clinical pathway (figure 4).

This mapping signifies a departure from a traditional clinical pathway, providing information on all treatment options early, and identifying patients' priorities and preferences to inform MDT recommendations in advance of the oncology consultation. The data confirm that a future intervention should include a 


\begin{tabular}{|c|c|c|c|c|c|c|}
\hline Gender & Age & MDT recommendation & Decided & Duration & Survival & Status \\
\hline Female & 72 & Pall chemo & Pall chemo & No treatment & 2 weeks & Deceased \\
\hline Female & 73 & Pall chemo & Pall chemo & Stopped & 3 weeks & Deceased \\
\hline Female & 71 & Pall chemo & Pall chemo & 3 of 4 cycles & 4 months & Deceased \\
\hline Female & 67 & Pall radio/chemo & Pall radio/no chemo & No chemo & 1 year & Deceased \\
\hline Female & 61 & Pall chemo/best supportive care & Best supportive care & No treatment & 4 weeks & Deceased \\
\hline Female & 52 & Pall chemo & Pall chemo & Completed & 3 months & Deceased \\
\hline Female & 65 & Pall chemo & Pall chemo & Stopped & 6 weeks & Deceased \\
\hline Male & 59 & Pall chemo & Pall chemo & Completed & 3 months & Alive* \\
\hline Male & 70 & Pall radio & Pall radio & Completed & 9 months & Alive $^{*}$ \\
\hline Male & 80 & Pall chemo/best supportive care & Best supportive care & No chemo & 3 months & Deceased \\
\hline Male & 76 & Pall chemo/trial & Pall chemo & 4 cycles & 1 month & Deceased \\
\hline Male & 66 & Pall chemo/trial & Pall chemo & 4 cycles & 5 months & Deceased \\
\hline Male & 68 & Pall chemo & Pall chemo & 4 cycles then pall radio after 1 year & 1 year/1 month & Deceased \\
\hline Male & 71 & Pall chemo & No treatment & No treatment & 4 months & Deceased \\
\hline Male & 65 & Pall chemo/trial & Trial & Ongoing & 3 months & Alive \\
\hline
\end{tabular}

${ }^{*}$ At end of study.

MDT, multidisciplinary team.

systematic collection and bidirectional flow of relevant information along the pathway, starting from primary care.

\section{DISCUSSION}

Tailored qualitative methods were used to understand how palliative chemotherapy treatment decisions are determined in advanced lung cancer. Data collection began with the MDT meetings where treatment discussions begin. MDT members constructed treatment recommendations mainly around patient performance status, pathology, clinical information and imaging. Patients' needs were not fully addressed because information about their social needs and preferences was limited. ${ }^{25}$

Measures of performance status did not fully capture patients' overall function and in this context where risks and benefits are finely balanced, alternative indicators regarding patients' ability to tolerate treatment, for example, frailty, cachexia should be considered. ${ }^{26-28}$ Here, the palliative care ethos of keeping people as

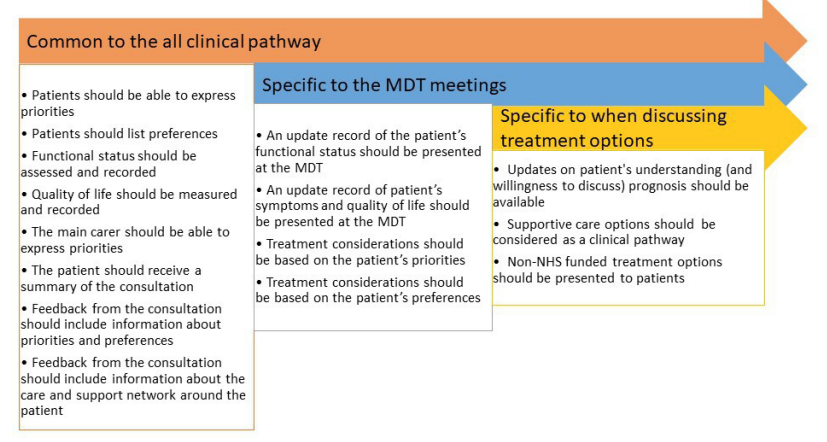

Figure 4 Statements of suggested practice mapped to the clinical pathway. MDT, multidisciplinary team; NHS, National Health Service. well as possible for as long as possible highlights the risks of tipping the balance-between preserving patients' function, and ability to take part in everyday activities, to hastening decline from avoidable toxicities. With increasing evidence to support the benefits of specialist palliative care for patients with advanced cancer, ${ }^{29}$ there is an argument against avoidable harm by SACT, and disenfranchisement of patients' access to better care.

Prognosis was the link for the oncologist to discuss available treatment options, and was compromised either by situations where a patient expressed a clear desire not to refer explicitly to prognosis, or where there was lack of information sharing among clinicians about how prognosis had been interpreted by the patient. ${ }^{30}$ Further, for patients with NSCLC, decisionmaking is a continuous process ${ }^{17}$ rather than a single event. With a rapid disease trajectory, the health of the patient might change significantly before the oncology appointment. The oncologist may have to re-evaluate the patient and consider a different treatment option to that recommended at the MDT. ${ }^{31}$ This proved particularly demanding for less experienced clinicians; training protocols have been developed but they did not always result in increased competency. ${ }^{32}$ Clinicians' attitude towards hope is also contributory to limited disclosure as Atul Gawande 33 describes it "Suppose I was wrong, I wondered, and she proved to be that miracle patient who survived metastatic lung cancer?" (p168). In addition to this, clinicians might be hesitant to crush patients' expectations when discussing palliative cancer treatments. Communication styles ${ }^{34} 35$ can affect patients' experiences and treatment choices, new approaches to treatment decisions, such as the global Choosing Wisely initiative ${ }^{36}$ encourage the use of prompt questions by clinicians and patients to 
weigh up the risks and benefits of treatment including the potential trigger question, 'What if I do nothing'.

Issues relating to palliative terminology often arose; ${ }^{4}$ however, patients' understanding of 'palliative' can be improved $^{37}$ and, differentiating between palliative care and end-of-life care, to what is effectively supportive oncology, could also improve referral. ${ }^{38}$ From a population perspective, societal challenges have been identified, including a public acceptance of death as part of being human, and the education of patients and families about treatment goals for cancer. ${ }^{39} 40$

The study adopted a coproductive approach, which ensured that all relevant stakeholders contributed to inform the intervention. The robust multiqualitative design allowed the study of the patient journey from different perspectives and levels. Patients represented geographical, and socioeconomic diversity, and different communities of professional practice. This study did not aim to contest or verify the appropriateness of the treatment decisions made. However, from the analysis of the treatment decision-making process, it emerged that there is a series of missed opportunities along the clinical pathway and these impacted on both clinicians and patients. Embedding patients' values and preferences into the treatment decision-making process may facilitate difficult consultations, reduce aggressive end-of-life treatments, and reflect more closely patients' goals and motives in their everyday lives.

\section{CONCLUSIONS}

Current cancer clinical pathways are not designed to include the circumstances, priorities and preferences of patients taking part in these highly sensitive consultations. The optimal management of advanced patients with NSCLC necessitates going beyond the treatment of symptoms by reflecting what matters to the patients within their personal context.

This patient population is characterised by a complex intersection of terminal disease with a short prognosis and declining functioning. Decisions around treatment towards end of life have a particular acuity, the limited patient understanding of the aims of treatment and the challenges faced by the clinicians who might resort to the treatment imperative may result in a clinical pathway heavily weighted towards SACT.

In the palliative context, the concept of hope realigns more realistically from hope of a cure to hope of comfort and quality of life. This study promotes opportunities for engagement with the patient (and family) and clinicians at different levels of the pathway to identify goals of care echoing the patient's view of a life worth living.

Although these findings reflect the narratives of the study participants and the researchers' interpretation of these, they also reflect elements easily applicable to many patients diagnosed with advanced cancer.
Twitter Annmarie Nelson @annmarienelson0 and Mirella Longo@MirellaLongo18

Contributors AN conceived and designed the study; drafted the manuscript. AN, SN, AB, SS and JL secured funding for the study. DA, CS and ML led the collection and analysis of the data. All authors made substantial contribution to the analysis and interpretation of the data. The public contributors (LR and DJ) supported the study throughout and carried out the validation of the findings. ML and CS contributed to the drafting of the manuscript. All authors reviewed and commented on the drafts of this manuscript. All authors read and approved the final manuscript. CS and DA are joint last authors.

Funding This study was funded by The Velindre Stepping Stones Appeal within Velindre NHS Trust Charitable Funds; Grant No 2013/009. Sponsored by Cardiff University; and coordinated by the Marie Curie core-funded Marie Curie Palliative Care Research Centre at Cardiff University. AN, AB, ML, SS and SN's posts are funded by Marie Curie Cancer Care core grant funding (grant reference MCCC-FCO-17-C).

Competing interests None declared.

Patient consent for publication Not required.

Ethics approval Ethical approval was granted (REC 14/ WA/1103).

Provenance and peer review Not commissioned; internally peer reviewed.

Data availability statement Data are available upon reasonable request. Anonymised narratives from the transcripts will be available upon request.

Open access This is an open access article distributed in accordance with the Creative Commons Attribution Non Commercial (CC BY-NC 4.0) license, which permits others to distribute, remix, adapt, build upon this work noncommercially, and license their derivative works on different terms, provided the original work is properly cited, appropriate credit is given, any changes made indicated, and the use is noncommercial. See: http://creativecommons.org/licenses/by-nc/4. $0 /$.

\section{ORCID iDs}

Mirella Longo http://orcid.org/0000-0002-9867-3806

Simon Noble http://orcid.org/0000-0001-5425-2383

\section{REFERENCES}

1 Garon EB, Hellmann MD, Rizvi NA, et al. Five-year overall survival for patients with advanced non-small-cell lung cancer treated with pembrolizumab: results from the phase I KEYNOTE-001 study. J Clin Oncol 2019;37:2518-27.

2 UK CR. Lung cancer statistics. secondary lung cancer statistics, 2014. Available: http://www.cancerresearchuk.org/healthprofessional/cancer-statistics/statistics-by-cancer-type/lungcancer/incidence\#heading-Three

3 Bluhm M, Connell CM, De Vries RG, et al. Paradox of prescribing late chemotherapy: oncologists explain. J Oncol Pract 2016;12:e1006-15.

4 McIlfatrick S, Noble H, McCorry NK, et al. Exploring public awareness and perceptions of palliative care: a qualitative study. Palliat Med 2014;28:273-80.

5 Weeks JC, Catalano PJ, Cronin A, et al. Patients' expectations about effects of chemotherapy for advanced cancer. N Engl J Med 2012;367:1616-25.

6 Khoja L, McGurk A, O'Hara C, O'Hara C, et al. Mortality within 30 days following systemic anti-cancer therapy, a review of all cases over a 4 year period in a tertiary cancer centre. Eur J Cancer 2015;51:233-40.

7 Mort D. For better for worse?: a review of the care of patients who died within 30 days of receiving systemic anti-cancer therapy. NCEPOD, 2008. 
8 O'Brien MR, Whitehead B, Murphy PN, et al. Social services homecare for people with motor neurone disease/amyotrophic lateral sclerosis: why are such services used or refused? Palliat Med 2012;26:123-31.

9 Yoong J, Seah J-A, Hamilton K, et al. Mortality within 30 days of receiving systemic anti-cancer therapy at a regional oncology unit: what have we learned? Asia Pac J Clin Oncol 2012;8:325-9.

10 Wallington M, Saxon EB, Bomb M, et al. 30-day mortality after systemic anticancer treatment for breast and lung cancer in England: a population-based, observational study. Lancet Oncol 2016;17:1203-16.

11 Earle CC, Neville BA, Landrum MB, et al. Trends in the aggressiveness of cancer care near the end of life. J Clin Oncol 2004;22:315-21.

12 Prigerson HG, Bao Y, Shah MA, et al. Chemotherapy use, performance status, and quality of life at the end of life. JAMA Oncol 2015;1:778-84.

13 Burgers JA, Damhuis RA. 30-Day mortality after the start of systemic anticancer therapy for lung cancer: is it really a useful performance indicator? ERJ Open Res 2018;4:000302018-2018.

14 Murray SA, Kendall M, Mitchell G, et al. Palliative care from diagnosis to death. BMJ 2017;356:j878.

15 Higginson IJ, Sen-Gupta GJ. Place of care in advanced cancer: a qualitative systematic literature review of patient preferences. J Palliat Med 2000;3:287-300.

16 Randén M, Helde-Frankling M, Runesdotter S, et al. Treatment decisions and discontinuation of palliative chemotherapy near the end-of-life, in relation to socioeconomic variables. Acta Oncol 2013;52:1062-6.

17 Traeger L, Rapoport C, Wright E, et al. Nature of discussions about systemic therapy discontinuation or hospice among patients, families, and palliative care clinicians during care for incurable cancer: a qualitative study. J Palliat Med 2020;23:542-7.

18 Greer JA, Pirl WF, Jackson VA, et al. Effect of early palliative care on chemotherapy use and end-of-life care in patients with metastatic non-small-cell lung cancer. J Clin Oncol 2012;30:394-400.

19 Wright AA, Zhang B, Ray A, et al. Associations between end-of-life discussions, patient mental health, medical care near death, and caregiver bereavement adjustment. JAMA 2008;300:1665-73.

20 Mack JW, Walling A, Dy S, et al. Patient beliefs that chemotherapy may be curative and care received at the end of life among patients with metastatic lung and colorectal cancer. Cancer 2015;121:1891-7.

21 Frenkel M. Refusing treatment. Oncologist 2013;18:634-6.

22 WHO. Framework on integrated, people-centred health services. 69th World Health Assembly provisional agenda item, 2016.

23 Anagnostou D, Sivell S, Noble S, et al. Development of an intervention to support patients and clinicians with advanced lung cancer when considering systematic anticancer therapy: protocol for the PACT study. BMJ Open 2017;7:e015277.
24 Elwyn G, Hutchings H, Edwards A, et al. The option scale: measuring the extent that clinicians involve patients in decision-making tasks. Health Expect 2005;8:34-42.

25 Soukup T, Lamb BW, Arora S, et al. Successful strategies in implementing a multidisciplinary team working in the care of patients with cancer: an overview and synthesis of the available literature. J Multidiscip Healthc 2018;11:49-61.

26 Rockwood K, Song X, MacKnight C, et al. A global clinical measure of fitness and frailty in elderly people. CMAJ 2005;173:489-95.

27 Cesari M, Gambassi G, van Kan GA, Abellan van Kan $\mathrm{G}$, et al. The frailty phenotype and the frailty index: different instruments for different purposes. Age Ageing 2014;43:10-12.

28 Moffatt H, Moorhouse P, Mallery L, et al. Using the frailty assessment for care planning tool (fact) to screen elderly chronic kidney disease patients for frailty: the nurse experience. Clin Interv Aging 2018;13:843-52.

29 Ziegler LE, Craigs CL, West RM, et al. Is palliative care support associated with better quality end-of-life care indicators for patients with advanced cancer? A retrospective cohort study. BMJ Open 2018;8:e018284.

30 Pfeil TA, Laryionava K, Reiter-Theil S, et al. What keeps oncologists from addressing palliative care early on with incurable cancer patients? an active stance seems key. Oncologist 2015;20:56-61.

31 Back AL, Trinidad SB, Hopley EK, et al. Reframing the goals of care conversation: "we're in a different place". J Palliat Med 2014;17:1019-24.

32 Johnson LA, Gorman C, Morse R, et al. Does communication skills training make a difference to patients' experiences of consultations in oncology and palliative care services? Eur J Cancer Care 2013;22:202-9.

33 Gawande A. Being mortal: illness, medicine, and what matters in the end. London, 2014.

34 Henselmans I, Van Laarhoven HW, Van der Vloodt J, et al. Shared decision making about palliative chemotherapy: a qualitative observation of talk about patients' preferences. Palliat Med 2017;31:625-33.

35 Kvåle K, Synnes O. Living with life-prolonging chemotherapycontrol and meaning-making in the tension between life and death. Eur J Cancer Care 2018;27:e12770.

36 Levinson W, Kallewaard M, Bhatia RS, et al. 'Choosing wisely': a growing international campaign. BMJ Qual Saf 2015;24:167-74.

37 Kozlov E, Reid MC, Carpenter BD. Improving patient knowledge of palliative care: a randomized controlled intervention study. Patient Educ Couns 2017;100:1007-11.

38 Temel JS, Greer JA, El-Jawahri A, et al. Effects of early integrated palliative care in patients with lung and Gi cancer: a randomized clinical trial. J Clin Oncol 2017;35:834-41.

39 Islam I, Byrne A, Nelson A. 27 Understanding public attitudes: a crucial component for developing palliative care services. BMJ Support Palliat Care 2018;8:370.1-370.

40 Harrington SE, Smith TJ. The role of chemotherapy at the end of life: "when is enough, enough?". JAMA 2008;299:2667-78. 